\title{
An Analysis of the Vector Decomposition Problem
}

\author{
Steven D. Galbraith ${ }^{1}$ and Eric R. Verheul ${ }^{2}$ \\ 1 Mathematics Department, \\ Royal Holloway, University of London, \\ Egham, Surrey, TW20 0EX, \\ United Kingdom \\ steven.galbraith@rhul.ac.uk \\ 2 PricewaterhouseCoopers Advisory, Radboud University Nijmegen, \\ P.O. Box 22735, 1100 DE, Amsterdam, The Netherlands \\ eric.verheul@\{nl.pwc.com, cs.ru.nl $\}$
}

\begin{abstract}
The vector decomposition problem (VDP) has been proposed as a computational problem on which to base the security of public key cryptosystems. We give a generalisation and simplification of the results of Yoshida on the VDP. We then show that, for the supersingular elliptic curves which can be used in practice, the VDP is equivalent to the computational Diffie-Hellman problem $(\mathrm{CDH})$ in a cyclic group. For the broader class of pairing-friendly elliptic curves we relate VDP to various co-CDH problems and also to a generalised discrete logarithm problem 2 -DL which in turn is often related to discrete logarithm problems in cyclic groups.
\end{abstract}

Keywords: Vector decomposition problem, elliptic curves, Diffie-Hellman problem, generalised discrete logarithm problem.

\section{Introduction}

The vector decomposition problem (VDP) is a computational problem in noncyclic groups $G$ (see Section 2 for the definition of this problem). It was introduced by Yoshida $22 \mid 23$ as an alternative to the discrete logarithm or DiffieHellman problems for the design of cryptographic systems. Yoshida proved that if certain conditions hold then the VDP is at least as hard as the computational Diffie-Hellman problem (CDH) in a certain cyclic subgroup $G_{1}$ of $G$. Since the $\mathrm{CDH}$ in $G_{1}$ may be hard, it follows that VDP may be hard, and so it is a potentially useful problem on which to base public key cryptography. Indeed, cryptosystems based on the VDP have been proposed in [22/2310.

As with any new computational problem in cryptography, it is important to understand the hardness of VDP if one is to use it in practice. Apart from the result of Yoshida, there is no discussion in the literature of the difficulty of the VDP. Hence, it is an open problem to determine the precise security level of the VDP and thus to evaluate the security/performance of cryptosystems based on it. That is the primary motivation of this paper. 
We prove that the VDP in $G$ is equivalent with certain co- $\mathrm{CDH}$ problems in $G$ if a mild condition holds. A corollary is that $\mathrm{CDH} \leq \mathrm{VDP}$ for a much larger class of groups than considered by Yoshida. We then prove that VDP $\leq \mathrm{CDH}$ for groups satisfying a condition similar to that considered by Yoshida (namely, existence of what we call a "distortion eigenvector base"). We show that all the supersingular elliptic curves which can be used in practice satisfy this condition. It follows that CDH and VDP are equivalent in practice for supersingular curves. We also prove this equivalence for the non-supersingular genus 2 curves proposed by Duursma and Kiyavasch [9]. Our results therefore completely resolve the issue of the difficulty of the VDP in the groups considered by 2223910 .

Duursma and Park [10] proposed a signature scheme based on VDP. Our results imply that their signature scheme has no security advantages over systems based on CDH or DLP. One can therefore compare the performance of the scheme in [10] with, say, Schnorr signatures and deduce that their scheme has no advantages in practice.

To summarise the paper: the main definitions and results are in Section 2 Section 3 proves that distortion eigenvector bases exist for the supersingular elliptic curves which can be used in practice. Section 4 explains how our conditions relate to the definitions given by Yoshida. In Section 5 we review possible constructions of non-cyclic groups for cryptography. Finally, Section 6 gives some methods to reduce the VDP to various generalised discrete logarithm problems.

\section{The Vector Decomposition Problem and Relations with CDH}

Let $r>3$ be a prime. The vector decomposition problem is usually expressed in terms of a 2-dimensional vector space over $\mathbb{F}_{r}$. However, it has currently only been instantiated on subgroups of exponent $r$ of the divisor class group of a curve over a finite field. Hence, in this paper we use a group-theoretic formulation.

Throughout the paper $G$ will be an abelian group of exponent $r$ and order $r^{2}$ (i.e., $G$ is isomorphic to $\left.(\mathbb{Z} / r \mathbb{Z}) \times(\mathbb{Z} / r \mathbb{Z})\right)$. We assume implicitly that $G$ can be represented compactly and that the group operation can be computed in polynomial time. For examples of such groups see Section 5. We write such groups additively and use capital letters $P, Q, R$ for elements of $G$. We use the notation $\left\langle P_{1}, \ldots, P_{n}\right\rangle$ for the subgroup of $G$ generated by $\left\{P_{1}, \ldots, P_{n}\right\}$. We call a pair $\left(P_{1}, P_{2}\right)$ a base for $G$ if it generates $G$, i.e. each element in $Q \in G$ can be uniquely written as a linear combination in $P_{1}$ and $P_{2}$.

If $\mathrm{A}$ and $\mathrm{B}$ are computational problems then we denote Turing reduction of $\mathrm{A}$ to $\mathrm{B}$ by $\mathrm{A} \leq \mathrm{B}$. This means that there is a polynomial time algorithm for solving problem A given access to an oracle to solve problem B. We call such a reduction tight if the probability of success of algorithm $\mathrm{A}$ is at least the probability of success of oracle B.

Definition 1. The vector decomposition problem (VDP): given a base $\left(P_{1}, P_{2}\right)$ for $G$ and an element $Q \in G$, compute an element $R \in G$ such that $R \in\left\langle P_{1}\right\rangle$ and $Q-R \in\left\langle P_{2}\right\rangle$. 
For a fixed base $\left(P_{1}, P_{2}\right)$ we define $V D P_{\left(P_{1}, P_{2}\right)}$ as: given $Q \in G$ find $R$ as above.

Clearly, such an element $R$ is unique and if we write $Q=a P_{1}+b P_{2}$ for unique $a, b \in \mathbb{Z} / r \mathbb{Z}$ then $R=a P_{1}$. We stress that an algorithm to solve the vector decomposition problem should take as input a triple $\left(P_{1}, P_{2}, Q\right)$ and output a point $R$ such that $R \in\left\langle P_{1}\right\rangle$ and $Q-R \in\left\langle P_{2}\right\rangle$. The VDP conjecture is that there exist families of groups for which the VDP is hard in the sense that there is no polynomial time algorithm which succeeds in solving the VDP on groups in the family with non-negligible probability over all possible input triples.

Yoshida proved that $\mathrm{CDH} \leq \mathrm{VDP}$ under certain conditions (see below). This suggests that VDP can be a hard problem. Our main goal in this paper is to give results in the other direction. As pointed out by an anonymous referee, an easy example of such a result can be obtained in the direct product of a cyclic group.

Definition 2. Let $G_{1}$ be a cyclic group of order $r$. The computational DiffieHellman problem $C D H\left(G_{1}\right)$ is: given $P, a P, b P \in G_{1}$, compute abP.

Lemma 1. Let $G_{1}$ be a cyclic group of prime order $r$ and let $G=G_{1} \times G_{1}$. If one can solve the VDP in $G$ then one can solve $C D H$ in $G_{1}$.

Proof. Let $P, a P, b P$ be the input CDH problem. Let $P_{1}=(P, a P), P_{2}=(0, P)$ and $Q=(b P, r P)$ for a random integer $r$. Note that $Q=b P_{1}+(r-a b) P_{2}$ so solving the $\operatorname{VDP}$ instance $\left(P_{1}, P_{2}, Q\right)$ gives $R=b P_{1}=(b P, a b P)$ and extracting the second component solves $\mathrm{CDH}$.

The literature on the VDP seems to contain only three examples of suitable groups. Precisely, Yoshida [23] suggests the supersingular elliptic curve $y^{2}=$ $x^{3}+1$ (see Example 1 below) and Duursma-Kiyavash 9] suggest two nonsupersingular genus 2 curves. However, it is obvious that one could use any pairing-friendly elliptic curve for applications based on the VDP.

We remark that VDP does not seem to trivially be random self-reducible. In other words, if we have an algorithm $\mathcal{A}$ which solves VDP for some non-negligible proportion of instances then it is not trivial to convert $\mathcal{A}$ into an algorithm which solves VDP with overwhelming probability over all instances. However, we show in Corollary 2 that one can obtain random self-reducibility for the VDP.

The following definition is the key concept which underlies most of the results in the paper.

Definition 3. Let $G$ be a group of exponent $r$ and order $r^{2}$. Let $F: G \rightarrow G$ be a group isomorphism computable in polynomial time. A pair of elements $S, T \in G$ is an eigenvector base with respect to $F$ if $G=\langle S, T\rangle$ and if $F(S)=\lambda_{1} S$ and $F(T)=\lambda_{2} T$ for some distinct, non-zero $\lambda_{1}, \lambda_{2} \in \mathbb{Z} / r \mathbb{Z}$.

In practice $F$ will usually be the Frobenius map (more details are given later). Hence we often abbreviate 'eigenvector base with respect to $F$ ' by 'eigenvector base'. 
Example 1. A standard example of such a group is as follows: Let $p \equiv 3 \quad(\bmod 4)$ be prime and let $E: y^{2}=x^{3}+x$ over $\mathbb{F}_{p}$. Then $E$ is a supersingular elliptic curve and $\# E\left(\mathbb{F}_{p}\right)=p+1$. Let $r>3$ be a prime such that $r \mid(p+1)$. Then we can let $G=E[r] \subseteq E\left(\mathbb{F}_{p^{2}}\right)$ be the group of all points on $E$ of order $r$. Let $S$ be a generator for $E\left(\mathbb{F}_{p}\right)[r]$. Denote by $F$ the $p$-power Frobenius map $F(x, y)=\left(x^{p}, y^{p}\right)$. Note that $F(S)=S$ so $\lambda_{1}=1$. Consider the isomorphism $\phi$ defined by $\phi(x, y)=(x, i y)$ where $i \in \mathbb{F}_{p^{2}}$ satisfies $i^{2}=-1$. Setting $T=\phi(S)$ we have $G=\langle S, T\rangle$ and $F(T)=-T$. Hence $(S, T)$ is an eigenvector base with respect to $F$. (Indeed, this is also a distortion eigenvector base, which will be defined later.)

Proposition 1. The $V D P_{\left(P_{1}, P_{2}\right)}$ with respect to a fixed base $\left(P_{1}, P_{2}\right)$ is solvable in polynomial time iff $\left(P_{1}, P_{2}\right)$ is an eigenvector base.

Proof. For the proof of the "if" part of the result: let $F: G \rightarrow G$ be the group isomorphism as in the definition of eigenvector base. Let $\alpha=\left(\lambda_{2}-\lambda_{1}\right)^{-1}$ $(\bmod r)$. For $i=1,2$ define the projection map $\psi_{i}: G \rightarrow\left\langle P_{i}\right\rangle$ by

$$
\psi_{1}(R)=\alpha\left(\lambda_{2} R-F(R)\right) ; \psi_{2}(R)=\alpha\left(F(R)-\lambda_{1} R\right) .
$$

These are efficiently computable group homomorphisms. Note that $\psi_{1}\left(P_{1}\right)=P_{1}$ and $\psi_{1}\left(P_{2}\right)=0$ and so $\psi_{1}$ maps to $\left\langle P_{1}\right\rangle$. Similarly, $\psi_{2}$ maps to $\left\langle P_{2}\right\rangle$. Since $Q=\psi_{1}(Q)+\psi_{2}(Q)$ for all $Q \in G$ and the maps $\psi_{1}, \psi_{2}$ are easily computable, it follows that VDP with respect to $\left(P_{1}, P_{2}\right)$ is easily solvable.

For the proof of the "only if" part of the result: suppose $\mathcal{A}$ is a polynomial time algorithm to solve $\operatorname{VDP}_{\left(P_{1}, P_{2}\right)}$. Define

$$
\psi_{1}(Q)=\mathcal{A}(Q) \text { and } \psi_{2}(Q)=Q-\psi_{1}(Q)
$$

Then $\psi_{i}(i=1,2)$ are group homomorphisms to $\left\langle P_{i}\right\rangle$ which can be computed in polynomial time. Any linear combination $F=\lambda \psi_{1}+\lambda_{2} \psi_{2}$ with distinct, nonzero $\lambda_{1}, \lambda_{2} \in \mathbb{Z} / r \mathbb{Z}$ has the desired properties so that $\left(P_{1}, P_{2}\right)$ is an eigenvector base.

The fact that there are easy instances of $\operatorname{VDP}_{\left(P_{1}, P_{2}\right)}$ does not affect the VDP conjecture for such curves. The conjecture is that the VDP should be hard for a randomly chosen input triple from the set $G^{3}$. In other words, it is permitted that the VDP be easy for a negligible proportion of triples in $G^{3}$.

\subsection{Diffie-Hellman Problems and Relation with VDP}

We recall the co-CDH problem as defined by Boneh, Lynn and Shacham [5].

Definition 4. Let $G_{1}$ and $G_{2}$ be cyclic groups of order $r$. The co-Computational Diffie-Hellman problem co-CDH $\left(G_{1}, G_{2}\right)$ is: Given $P, a P \in G_{1}$ and $Q \in G_{2}$, compute $a Q$.

Note that having a perfect algorithm to solve co- $\mathrm{CDH}$ is equivalent to being able to compute a group homomorphism $\psi: G_{1} \rightarrow G_{2}$ such that $\psi(P)=Q$. 
Lemma 2. Let $G_{1}, G_{2}$ be cyclic groups of order $r$. Then $C D H\left(G_{1}\right) \leq($ co$\mathrm{CDH}\left(G_{1}, G_{2}\right)$ and $\left.\mathrm{co}-\mathrm{CDH}\left(G_{2}, G_{1}\right)\right)$.

Proof. Suppose we have oracles to solve both co-CDH problems which succeed with probability at least $\epsilon$. Let $P, a P, b P$ be given. Choose a random $Q \in G_{2}$ and a random $x \in(\mathbb{Z} / r \mathbb{Z})^{*}$ and call the co- $\mathrm{CDH}\left(G_{1}, G_{2}\right)$ oracle on $(x P, x a P, Q)$ to get $a Q$ with probability at least $\epsilon$.

Now, choose random $x_{1}, x_{2} \in(\mathbb{Z} / r \mathbb{Z})^{*}$ and call the co- $\mathrm{CDH}\left(G_{2}, G_{1}\right)$ oracle on $\left(x_{1} Q, x_{1} a Q, x_{2} b P\right)$ to get $x_{2} a b P$ with probability at least $\epsilon$. Exponentiating by $x_{2}^{-1}$ gives $a b P$ as desired. The probability of success is at least $\epsilon^{2}$.

In Lemma 4 we give a converse to the above result if additional conditions hold (e.g., for supersingular elliptic curves). Note that if one can solve $\mathrm{CDH}\left(G_{1}\right)$ and one has a suitable auxiliary elliptic curve for the Maurer reduction [15]16 then one can solve the DLP in $G_{1}$ and hence solve co- $\mathrm{CDH}\left(G_{1}, G_{2}\right)$. Hence it is natural to conjecture that $\operatorname{CDH}\left(G_{1}\right)$ and $\operatorname{co}-\mathrm{CDH}\left(G_{1}, G_{2}\right)$ are equivalent. However, it could conceivably be the case that there exist groups such that (co-CDH $\left(G_{1}, G_{2}\right)$ and co- $\left.\operatorname{CDH}\left(G_{2}, G_{1}\right)\right)$ is strictly harder than $\operatorname{CDH}\left(G_{1}\right)$. It would follow from Theorem 1 below that VDP is a strictly harder problem than $\operatorname{CDH}\left(G_{1}\right)$ for these groups.

The following computational problem is similar to the problem DCDH defined by Bao et al 2, who also proved equivalence with $\mathrm{CDH}$. For completeness we give a trivial Lemma which is needed later.

Definition 5. The co-Divisional Computational Diffie-Hellman problem co-DCDH $\left(G_{1}, G_{2}\right)$ is, given $(S, a S, T)$ for $S \in G_{1}, T \in G_{2}$, to compute $a^{-1} T$.

Lemma 3. co- $D C D H\left(G_{1}, G_{2}\right) \leq \operatorname{co-} C D H\left(G_{1}, G_{2}\right)$.

Proof. Given a co-DCDH instance $(S, a S, T)$ choose uniformly at random $x_{1}, x_{2}$, $x_{3} \in(\mathbb{Z} / r \mathbb{Z})^{*}$ and return $\left(x_{2} x_{3}\right)^{-1} \operatorname{co}-\mathrm{CDH}\left(x_{1} a S, x_{1} x_{2} S, x_{3} T\right)$. Hence, if we can solve co-CDH with probability at least $\epsilon$ then one can solve co-DCDH with probability at least $\epsilon$.

Yoshida 22 23] showed that CDH $\leq$ VDP for supersingular elliptic curves having endomorphisms satisfying certain conditions. Theorem 1 below gives a major extension of Yoshida's result, since it has much weaker conditions and can be applied to ordinary curves (we give more discussion of this later). Also note that Yoshida's result requires a perfect oracle to solve VDP (i.e., one which always succeeds) whereas our proof allows an oracle with only some non-negligible probability of success (this is a non-trivial improvement since VDP does not seem to trivially have random self-reducibility).

Theorem 1. Let $G$ have an eigenvector base $(S, T)$ and define $G_{1}=\langle S\rangle, G_{2}=$ $\langle T\rangle$. Then VDP is equivalent to (co-CDH $\left(G_{1}, G_{2}\right)$ and co-CDH $\left(G_{2}, G_{1}\right)$ ).

More precisely, if one can solve VDP with probability at least $\epsilon$ then one can solve (co-CDH( $\left.G_{1}, G_{2}\right)$ and co- $\left.C D H\left(G_{2}, G_{1}\right)\right)$ with probability at least $\epsilon$. If one can solve (co-CDH( $\left.G_{1}, G_{2}\right)$ and co- $\left.C D H\left(G_{2}, G_{1}\right)\right)$ with probability at least $\epsilon$ then one can solve VDP with probability at least $\epsilon^{9}$. 
Proof. First we show that $\operatorname{co}-\mathrm{CDH}\left(G_{1}, G_{2}\right) \leq \mathrm{VDP}$ (the full statement follows by symmetry). We assume that we have a VDP oracle which succeeds with probability $\epsilon$ and show that one can solve co- $\mathrm{CDH}\left(G_{1}, G_{2}\right)$ with probability $\epsilon$.

Let $S, a S, T$ be given. Choose uniformly at random $x_{1}, x_{2}, y_{1}, y_{2} \in(\mathbb{Z} / r \mathbb{Z})$ such that $x_{1} x_{2}-y_{1} y_{2} \not \equiv 0 \quad(\bmod r)$. Then $\left(P_{1}=x_{1} S+y_{1} T, P_{2}=y_{2} S+x_{2} T\right)$ is a uniformly random base for $G$. There exist $\lambda, \mu \in(\mathbb{Z} / r \mathbb{Z})$ such that $a S=$ $\lambda P_{1}+\mu P_{2}$. One has

$$
a S=\lambda\left(x_{1} S+y_{1} T\right)+\mu\left(y_{2} S+x_{2} T\right)=\left(\lambda x_{1}+\mu y_{2}\right) S+\left(\lambda y_{1}+\mu x_{2}\right) T
$$

and so

$$
\left(\begin{array}{ll}
x_{1} & y_{2} \\
y_{1} & x_{2}
\end{array}\right)\left(\begin{array}{l}
\lambda \\
\mu
\end{array}\right)=\left(\begin{array}{l}
a \\
0
\end{array}\right) .
$$

Calling a VDP oracle on $\left(P_{1}, P_{2}, a S+u_{1} P_{1}+u_{2} P_{2}\right)$ for uniformly random $u_{1}, u_{2} \in(\mathbb{Z} / r \mathbb{Z})$ and subtracting $u_{1} P_{1}$ from the output gives $\lambda P_{1}=\lambda x_{1} S+\lambda y_{1} T$ with probability $\epsilon$. Using Proposition 1 one can compute $R=\lambda y_{1} T$.

Equation (10) implies that $\lambda \equiv\left(x_{1} x_{2}-y_{1} y_{2}\right)^{-1} x_{2} a \quad(\bmod r)$. It follows that one can compute $a T$ as

$$
a T=\left(x_{1} x_{2}-y_{1} y_{2}\right)\left(y_{1} x_{2}\right)^{-1} R
$$

This completes the first part of the proof.

For the second part, we assume oracles to solve co- $\mathrm{CDH}\left(G_{1}, G_{2}\right)$ and co$\operatorname{CDH}\left(G_{2}, G_{1}\right)$ which work with probability at least $\epsilon$. By Lemma 2 we can also solve ordinary $\mathrm{CDH}$ in $\langle S\rangle$ and $\langle T\rangle$ with probability at least $\epsilon^{2}$. We will show how to solve VDP with probability at least $\epsilon^{9}$.

Let $\left(P_{1}, P_{2}, Q\right)$ be the input instance of the VDP. Then

$$
Q=a P_{1}+b P_{2}
$$

for unknown integers $(a, b)$. Our goal is to compute $a P_{1}$.

There exist (unknown) integers $u_{i, j}$ for $1 \leq i, j \leq 2$ such that

$$
P_{i}=u_{1, i} S+u_{2, i} T
$$

and integers $\left(v_{1}, v_{2}\right)$ such that $Q=v_{1} S+v_{2} T$. By Proposition 1 we can compute $u_{1, i} S, u_{2, i} T, v_{1} S$ and $v_{2} T$.

Write

$$
U=\left(\begin{array}{ll}
u_{1,1} & u_{1,2} \\
u_{2,1} & u_{2,2}
\end{array}\right) .
$$

Since $\{S, T\}$ and $\left\{P_{1}, P_{2}\right\}$ both generate $G$, it follows that $U$ is invertible. Clearly,

$$
v_{1} S+v_{2} T=Q=a P_{1}+b P_{2}=\left(a u_{1,1}+b u_{1,2}\right) S+\left(a u_{2,1}+b u_{2,2}\right) T
$$

and so

$$
U\left(\begin{array}{l}
a \\
b
\end{array}\right)=\left(\begin{array}{l}
v_{1} \\
v_{2}
\end{array}\right) .
$$


Hence,

$$
\left(\begin{array}{l}
a \\
b
\end{array}\right)=\left(u_{1,1} u_{2,2}-u_{1,2} u_{2,1}\right)^{-1}\left(\begin{array}{cc}
u_{2,2} & -u_{1,2} \\
-u_{2,1} & u_{1,1}
\end{array}\right)\left(\begin{array}{l}
v_{1} \\
v_{2}
\end{array}\right)
$$

and so

$$
a P_{1}=\left(u_{1,1} u_{2,2}-u_{1,2} u_{2,1}\right)^{-1}\left(u_{2,2} v_{1}-u_{1,2} v_{2}\right)\left(u_{1,1} S+u_{2,1} T\right) .
$$

Compute $u_{2,2} v_{1} T, u_{1,1} u_{2,2} S$ and $u_{1,2} u_{2,1} S$ using 3 calls to co-CDH oracles and $u_{1,2} v_{2} T$ using one call to a $\mathrm{CDH}$ oracle for $\langle T\rangle$ (which is achieved using 2 calls to co-CDH oracles). Then solve co-DCDH $\left(S,\left(u_{1,1} u_{2,2}-u_{1,2} u_{2,1}\right) S,\left(u_{2,2} v_{1}-\right.\right.$ $\left.u_{1,2} v_{2}\right) T$ ) using Lemma 3 to get $a T$.

Given $S, u_{1,1} S, a T$ and $u_{2,1} T$ one can compute $a P_{1}$ with one call to a $\mathrm{CDH}$ oracle for $\langle T\rangle$ and one call to a co-CDH oracle. It follows that we require 5 co$\mathrm{CDH}$ queries and $2 \mathrm{CDH}$ queries, which means that the algorithm succeeds with probability at least $\epsilon^{9}$.

Corollary 1. Let $G$ be as above and suppose $G$ has an eigenvector base $(S, T)$. Let $G_{1}=\langle S\rangle$. Then $C D H\left(G_{1}\right) \leq V D P$.

More precisely, if one has an oracle to solve VDP with probability at least $\epsilon$ then one can solve $\mathrm{CDH}\left(G_{1}\right)$ with probability at least $\epsilon^{2}$.

Proof. This is immediate from Theorem 1 and Lemma 2

Corollary 2. Suppose $G$ has an eigenvector base. Then the VDP has random self-reducibility.

Proof. The second part of the proof of Theorem 1 shows how to convert a VDP instance into a number of co-CDH instances. The first part of the proof of Theorem 1 shows how to convert a co-CDH instance into a uniformly random instance of the VDP in $G$. Hence, a specific VDP instance in $G$ is reduced to a number of uniformly random VDP instances in $G$.

\subsection{Distortion Eigenvector Bases and Equivalence of VDP and $\mathrm{CDH}$}

Definition 6. An eigenvector base $(S, T)$ is said to be a distortion eigenvector base if there are group homomorphisms $\phi_{1}:\langle S\rangle \rightarrow\langle T\rangle$ and $\phi_{2}:\langle T\rangle \rightarrow\langle S\rangle$ computable in polynomial time and if an integer $d \not \equiv 0(\bmod r)$ is given such that $\phi_{2}\left(\phi_{1}(S)\right)=d S$.

In Section 3 we will show that the commonly used pairing-friendly supersingular elliptic curves all have a distortion eigenvector base.

Lemma 4. Let $G$ be as above and suppose $G$ has a distortion eigenvector base $(S, T)$. Let $G_{1}=\langle S\rangle$ and $G_{2}=\langle T\rangle$. Then $C D H\left(G_{1}\right)$ is equivalent to co$C D H\left(G_{1}, G_{2}\right)$ and co- $C D H\left(G_{2}, G_{1}\right)$. Moreover, the reductions in both directions are tight. 
Proof. Suppose we have an oracle to solve CDH with probability at least $\epsilon$. Given a co-CDH instance $(S, a S, T)$ we want to compute $a T$. Note that $\phi_{2}(T)=c S$ for some (not necessarily explicitly known) integer $c$ and that $\phi_{1}(c S)=d T$ for known $d$. Since $\operatorname{CDH}(S, a S, c S)=a c S$ it follows that the solution to the co-CDH problem is given by

$$
\left(d^{-1}(\bmod r)\right) \phi_{1}\left(\mathrm{CDH}\left(S, a S, \phi_{2}(T)\right)\right) .
$$

Hence, we can solve co-CDH with probability at least $\epsilon$ (note that $\mathrm{CDH}$ and co-CDH are clearly random self-reducible).

For the converse, suppose $S, a S, b S$ is an instance of $\operatorname{CDH}\left(G_{1}\right)$. Then one obtains the co-CDH instance $\left(S, a S, \phi_{1}(b S)\right)$ and the solution to the $\mathrm{CDH}$ is $\left(d^{-1}(\bmod r)\right) \phi_{2}\left(\operatorname{co}-\mathrm{CDH}\left(S, a S, \phi_{1}(b S)\right)\right)$.

This allows a refinement of Corollary 1.

Corollary 3. Suppose $G$ has a distortion eigenvector base $(S, T)$ and let $G_{1}=$ $\langle S\rangle$. Suppose one has an oracle to solve VDP with probability at least $\epsilon$. Then one can solve $\mathrm{CDH}\left(\mathrm{G}_{1}\right)$ with probability at least $\epsilon$.

We then obtain one of the main results in the paper, that VDP is equivalent to $\mathrm{CDH}$ in many cases. This is a significant sharpening of Yoshida's result, and gives a complete understanding of VDP for supersingular curves.

Corollary 4. Let $(S, T)$ be a distortion eigenvector base for $G$. Then VDP is equivalent to $C D H(\langle S\rangle)$.

Proof. Let $G_{1}=\langle S\rangle$ and $G_{2}=\langle T\rangle$. Theorem 1 showed VDP equivalent to $\operatorname{co}-\operatorname{CDH}\left(G_{1}, G_{2}\right)$ and $\operatorname{co}-\operatorname{CDH}\left(G_{2}, G_{1}\right)$ and so the result follows by Lemma 4 ,

Note that when given a CDH oracle then the probability of success in Theorem 1 is $\epsilon^{7}$ instead of $\epsilon^{9}$.

\subsection{An Application of Trapdoor VDP}

Proposition 1 shows that VDP is easy for certain bases while Theorem 11indicates that VDP is hard in general. Hence it is natural to ask if there is a way to set up a trapdoor VDP system. We now explain how to do this.

Proposition 2. Let $(S, T)$ be a distortion eigenvector base for $G$ normalised such that $T=\phi_{1}(S)$. Let $u_{1,1}, u_{1,2}, u_{2,1}, u_{2,2} \in \mathbb{Z} / r \mathbb{Z}$ be such that $u_{1,1} u_{2,2}-$ $u_{1,2} u_{2,1} \not \equiv 0 \quad(\bmod r)$. Let $P_{1}=u_{1,1} S+u_{2,1} T$ and $P_{2}=u_{1,2} S+u_{2,2} T$. Given any $Q \in G$, if one knows the $u_{i, j}$ then one can solve the VDP of $Q$ to the base $\left(P_{1}, P_{2}\right)$.

Proof. We have $T=\phi_{1}(S)$ and replacing $\phi_{2}$ by $\left(d^{-1}(\bmod r)\right) \phi_{2}$ we have $\phi_{2}(T)=S$.

Write $Q=a P_{1}+b P_{2}$. We are required to compute $a P_{1}$. Since $(S, T)$ is an eigenvector base we can compute $v_{1} S$ and $v_{2} T$ such that $Q=v_{1} S+v_{2} T$. Using $\phi_{1}$ 
and $\phi_{2}$ we can compute $v_{1} T$ and $v_{2} S$. By the same arguments as in Theorem 1 writing $w=\left(u_{1,1} u_{2,2}-u_{1,2} u_{2,1}\right)^{-1} \quad(\bmod r)$, it follows that

$$
\begin{aligned}
a P_{1} & =w\left(u_{2,2} v_{1}-u_{1,2} v_{2}\right)\left(u_{1,1} S+u_{2,1} T\right) \\
& =w\left(u_{2,2} u_{1,1} v_{1} S+u_{2,2} u_{2,1} v_{1} T-u_{1,1} u_{1,2} v_{2} S-u_{1,2} u_{2,1} v_{2} T\right)
\end{aligned}
$$

which is easily computed.

Note that we do not have a full trapdoor which allows solving any instance $\left(P_{1}, P_{2}, Q\right)$ of the VDP. Instead, we construct an easy base $\left(P_{1}, P_{2}\right)$ for the VDP from an existing easy base $(S, T)$.

This idea has several cryptographic applications. For example, one can obtain a public key encryption scheme (having OW-CPA security depending on VDP) with public key $\left(S, Q=u_{1,2} S+u_{2,2} T\right)$ and where the private key consists of the $u_{i, j}$. A message $M \in\langle S\rangle$ is encrypted as $C=M+b Q$ for random $1 \leq b<r$.

\subsection{The Decision Vector Decomposition Problem}

As suggested by an anonymous referee, one can consider a decision variant of the VDP.

Definition 7. The decision vector decomposition problem (DVDP) is: given $\left(P_{1}, P_{2}, Q, R\right)$ to test whether $R \in\left\langle P_{1}\right\rangle$ and $(Q-R) \in\left\langle P_{2}\right\rangle$.

Hence the DVDP is just testing subgroup membership, which is a computational problem in cyclic groups rather than in $G$ and which may or may not be easy depending on the groups in question. For example, if $G=E[r]$ for an elliptic curve then one can test subgroup membership using the Weil pairing (namely, $R \in\left\langle P_{1}\right\rangle$ if and only if $\left.e_{r}\left(P_{1}, R\right)=1\right)$. Also, if $(S, T)$ is an eigenvector base with respect to $F$ then testing subgroup membership is easy $(P \in\langle S\rangle$ if and only if $F(P)=\lambda_{1} P$ where $\lambda_{1}$ is the eigenvalue of $F$ on $\left.S\right)$.

The decision version of the co-CDH problem is defined as follows [5].

Definition 8. Let $G_{1}$ and $G_{2}$ be distinct cyclic groups of order $r$. The codecision Diffie-Hellman problem co-DDH $\left(G_{1}, G_{2}\right)$ is: Given $S, a S \in G_{1}$ and $T, T^{\prime} \in G_{2}$ to determine whether or not $T^{\prime}=a T$.

Note that co- $\operatorname{DDH}\left(G_{1}, G_{2}\right)$ is trivially equivalent to $\operatorname{co-} \operatorname{DDH}\left(G_{2}, G_{1}\right)$.

Lemma 5. If $G_{1}$ and $G_{2}$ are distinct cyclic subgroups of $G$ then co-DDH $\left(G_{1}, G_{2}\right)$ $\leq D V D P$ in $G$.

Proof. Suppose we have an oracle to solve DVDP and let $\left(S, a S, T, T^{\prime}\right)$ be the input co-DDH instance. We assume that $\langle S\rangle \cap\langle T\rangle=\{0\}$ and that $T^{\prime} \in\langle T\rangle$. Let $b \in(\mathbb{Z} / r \mathbb{Z})$ be such that $T^{\prime}=b T$.

Choose random $x_{1,1}, x_{1,2}, x_{2,1}, x_{2,2}, z \in(\mathbb{Z} / r \mathbb{Z})^{*}$ such that $x_{1,1} x_{2,2}-x_{1,1} x_{2,1} \not \equiv$ $0 \quad(\bmod r)$. Let $P_{1}=x_{1,1} S+x_{2,1} T, P_{2}=x_{1,2} S+x_{2,2} T, Q=x_{1,1} a S+x_{2,1} T^{\prime}+$ $z P_{2}$ and $R=x_{1,1} a S+x_{2,1} T^{\prime}$ and call the DVDP oracle on $\left(P_{1}, P_{2}, Q, R\right)$. If $b \equiv a$ $(\bmod r)$ then $R \in\left\langle P_{1}\right\rangle$ and the oracle should answer 'true'. If $b \neq \equiv a(\bmod r)$ then $R \notin\left\langle P_{1}\right\rangle$ and the oracle should answer 'false'. 
One can verify that for the case $G=E[r]$, where DVDP is easily solved using the Weil pairing, the proof of Lemma 5 leads to the standard method for solving co$\mathrm{DDH}$ using pairings (note that if $G_{1}$ and $G_{2}$ are distinct in $E[r]$ then $e_{r}(S, T) \neq 1$ ).

Theorem 2. Let $G$ have an eigenvector base $(S, T)$ and define $G_{1}=\langle S\rangle, G_{2}=$ $\langle T\rangle$. Then DVDP is equivalent to co-DDH $\left(G_{1}, G_{2}\right)$.

Proof. Lemma 5 gives co-DDH $\left(G_{1}, G_{2}\right) \leq$ DVDP. To prove the converse we show how to solve the subgroup membership problem for any subgroup $H=$ $\langle R\rangle \subset G$. If $H=\langle S\rangle$ or $H=\langle T\rangle$ then, as mentioned, we can efficiently solve membership. Hence, we may assume that the projections $\psi_{1}(R)$ and $\psi_{2}(R)$ in the proof of Proposition 1 are non-trivial. Let $P \in G$. Then $P \in\langle R\rangle$ if and only if $\left(\psi_{1}(R), \psi_{1}(P), \psi_{2}(R), \psi_{2}(P)\right)$ is a valid co-DDH $\left(G_{1}, G_{2}\right)$ instance. The result follows.

One might expect a version of the Theorem 2 without the requirement to have an eigenvector base. In fact, the ability to test subgroup membership (and hence solve DVDP) is essentially implicit in the statement of co-DDH: How does one know that $S, a S \in G_{1}$ and $T, T^{\prime} \in G_{2}$ ? What is the behaviour of a co-DDH oracle if any of these conditions does not hold?

\section{Existence of Distortion Eigenvector Bases}

We have shown that VDP is equivalent to $\mathrm{CDH}$ when $G$ has an distortion eigenvector base. The goal of this section is to show that all the supersingular elliptic curves used in practice have a distortion eigenvector basis. The restriction to "curves used in practice" is because for the case of elliptic curves over $\mathbb{F}_{p}$ we use an algorithm from 14 whose complexity is exponential in the class number $h$ of the CM field $\mathbb{Q}\left(\sqrt{t^{2}-4 p}\right)$. Although this algorithm has exponential complexity in general, it has polynomial complexity if the class number is bounded by a polynomial in $\log (p)$ (for the purposes of this paper let's insist that $h \leq \log (p)^{2}$ ). Hence the algorithm runs in polynomial time for all curves which can be constructed in polynomial time using the CM method (which is all supersingular curves used in practice) 1 See [14 for more discussion of this issue.

We summarise some standard examples of supersingular elliptic curves and distortion maps $\phi$ in Table 1 The triple $\left(\alpha_{1}, \alpha_{2}, \alpha_{3}\right)$ in the table means that for $S \in E\left(\mathbb{F}_{q}\right)$ and $\pi$ the $q$-power Frobenius map we have $\pi(S)=\alpha_{1} S$ and $\pi(\phi(S))=$ $\alpha_{2} S+\alpha_{3} \phi(S)$ (this is the notation of Yoshida [23]). Using Proposition 3 below we can obtain from the table the maps $\phi_{1}$ and $\phi_{2}$ required in Definition 6. Specifically, for the first row of Table 1 one can take (see Theorem 4 for details) $\phi_{1}=m+\phi$ and $\phi_{2}=m+\phi^{2}$ where $m \equiv 2^{-1} \quad(\bmod r)\left(\operatorname{giving} d \equiv m^{2}-m+1\right.$ $(\bmod r)$, where $d$ is such that $\left.\phi_{2}\left(\phi_{1}(S)\right)=d S\right)$, for the last row take $\phi_{1}=\phi$ and

${ }^{1}$ One can construct $E$ such that $\operatorname{End}(E)$ is not the maximal order in $\mathbb{Q}\left(\sqrt{t^{2}-4 p}\right)$. However, one can use isogenies to reduce to the case where $\operatorname{End}(E)$ is maximal, so throughout the paper we assume this is the case. 
Table 1. Suitable elliptic curves for the Yoshida conditions

\begin{tabular}{|c|c|c|c|c|}
\hline$E$ & $q$ & $k$ & $\phi(x, y)$ & $\left(\alpha_{1}, \alpha_{2}, \alpha_{3}\right)$ \\
\hline$y^{2}=x^{3}+1$ & $p$ & 2 & $\begin{array}{l}\left(\zeta_{3} x, y\right) \quad \text { where } \\
\zeta_{3}^{2}+\zeta_{3}+1=0\end{array}$ & $(1,-1,-1)$ \\
\hline$y^{2}=x^{3}+x$ & $\begin{array}{l}p \\
p \equiv 3 \quad(\bmod 4)\end{array}$ & 2 & \begin{tabular}{|ll}
$(-x, i y) \quad$ where \\
$i^{2}=-1$ & \\
\end{tabular} & $(1,0,-1)$ \\
\hline$y^{2}+y=x^{3}+x+b$ & $\begin{array}{l}2^{m} \\
\operatorname{gcd}(m, 2)=1\end{array}$ & 4 & $\begin{array}{l}\left(x+\zeta_{3}^{2}, y+\zeta_{3} x+t\right) \\
\zeta_{3}^{2}+\zeta_{3}+1=0, t^{2}+t=\zeta_{3}\end{array}$ & $(1,0,-1)$ \\
\hline$y^{2}=x^{3}-x+b$ & $\begin{array}{l}3^{m} \\
\operatorname{gcd}(m, 6)=1\end{array}$ & 6 & $\begin{array}{l}(\rho-x, i y) \quad \text { where } \\
\rho^{3}-\rho=b, i^{2}=-1\end{array}$ & $(1,0,-1)$ \\
\hline $\begin{array}{l}y^{2}=x^{3}+A \text { where } \\
A \in \mathbb{F}_{p^{2}} \text { is a square } \\
\text { but not a cube }\end{array}$ & $\begin{array}{l}p^{2} \\
p \equiv 2 \quad(\bmod 3)\end{array}$ & 3 & $\begin{array}{l}\left(\gamma^{2} x^{p}, u y^{p}\right) \quad \text { where } \\
u^{2}=A / A^{q}, u \in \mathbb{F}_{p^{2}} \\
\gamma^{3}=u, \gamma \in \mathbb{F}_{p^{6}}\end{array}$ & $\begin{array}{c}(1,0, \lambda) \quad \text { where } \\
\lambda^{2}+\lambda+1 \equiv 0 \\
(\bmod r)\end{array}$ \\
\hline
\end{tabular}

$\phi_{2}(x, y)=\left(\left(x / \gamma^{2}\right)^{p},(y / u)^{p}\right)$ (so $\left.d=1\right)$ and for the other three entries one can take $\phi_{1}=\phi_{2}=\phi($ so $d=-1)$. This shows that all the elliptic curves in Table 1 have a distortion eigenvector base.

A corollary of Theorem 3 below is that for every supersingular elliptic curve used in practice there are $(P, \phi, F)$ satisfying the Yoshida conditions. Recall that Duursma and Kiyavash showed that if $E$ is an elliptic curve over a finite field with a point $P$ and maps $\phi, F$ which satisfy the Yoshida conditions (see Section 4 below) then $E$ is supersingular. Hence our corollary gives a complete classification of elliptic curves used in practice satisfying the Yoshida conditions.

The restriction to supersingular curves is not surprising: If $E$ is an elliptic curve with a distortion eigenvector base and if $F$ and the group homomorphisms $\phi_{1}, \phi_{2}$ are endomorphisms of the elliptic curve, then $E$ must be supersingular ( $F$ and $\phi_{1}$ do not commute, so the endomorphism ring is non-commutative).

The case of embedding degree 1 is more subtle. Frobenius acts as the identity, so for an eigenvector base one must take $F$ to be an endomorphism which is not in $\mathbb{Z}[\pi]$ (where $\pi$ is the $q$-power Frobenius) but which has (at least) two eigenspaces. Such endomorphisms may or may not exist (see Charles [7]). Distortion eigenvector bases do not exist when $k=1$ since a further endomorphism is required which does not commute with $F$ or $\pi$, and for elliptic curves there can be no such maps.

We begin with three lemmas to deal with the case of embedding degree 3 (i.e., $r \mid \# E\left(\mathbb{F}_{q}\right)$ has $\left.r \mid\left(q^{3}-1\right)\right)$. For background in this section see 4819 ]

Lemma 6. Let $E$ be an elliptic curve over $\mathbb{F}_{q^{2}}$ with $\# E\left(\mathbb{F}_{q^{2}}\right)=q^{2} \pm q+1$. Then $j(E)=0$.

Proof. Let $\pi$ be the $q^{2}$-power Frobenius map, which has degree $q^{2}$ and is purely inseparable. Since $E$ is supersingular ( $q$ divides the trace of Frobenius) it follows that $[q]$ is also purely inseparable of degree $q^{2}$. Therefore (see Silverman [19] Corollary II.2.12), $[q]=\phi \pi$ where $\phi \in \operatorname{End}(E)$. Taking degrees implies that $\operatorname{deg}(\phi)=1$ and, since $\pi$ and $[q]$ are defined over $\mathbb{F}_{q^{2}}$, it follows that $\phi$ is also defined over $\mathbb{F}_{q^{2}}$ and so $\pi \phi=\phi \pi$. 
Substituting $q=\phi \pi$ into the characteristic polynomial of Frobenius gives

$$
0=\pi^{2} \pm q \pi+q^{2}=\left(\phi^{2} \pm \phi+1\right) \pi^{2}
$$

and hence the automorphism $\phi$ satisfies $\phi^{2} \pm \phi+1=0$. It follows that $\pm \phi \in$ $\operatorname{End}(E)$ is an automorphism of order 3. This implies (see [19] Theorem III.10.1) that $j(E)=0$.

Lemma 7. Let $E_{A}: y^{2}=x^{3}+A$ be an elliptic curve over $\mathbb{F}_{q^{2}}$ with $q=p^{m}$ such that $p>3$. Then $\# E_{A}\left(\mathbb{F}_{q^{2}}\right)=q^{2} \pm q+1$ if and only if $p \equiv 2(\bmod 3)$ and $A$ is not a cube.

Proof. We sketch the proof; see the full version of the paper for all the details.

It is a standard fact $[19$ that $E$ is supersingular if and only if $p \equiv 2 \quad(\bmod 3)$. Let $g$ be a primitive element of $\mathbb{F}_{q^{2}}$. Then $E_{A}$ is isomorphic over $\mathbb{F}_{p^{2}}$ to one of the curves $E_{g^{i}}: y^{2}=x^{3}+g^{i}$ for $0 \leq i<6$. We will determine which of these curves has $q^{2} \pm q+1$ points.

It is easy to check that $E_{1}: y^{2}=x^{3}+1$ over $\mathbb{F}_{q}$ has $q+1=p^{m}+1$ points if $m$ is odd, $\left(p^{d}+1\right)^{2}$ points if $m=2 d$ where $d$ is odd, and $\left(p^{d}-1\right)^{2}$ points if $m=2 d$ where $d$ is even. Hence the characteristic polynomial of Frobenius over $\mathbb{F}_{q^{2}}$ is $(T \pm q)^{2}$ and $\# E_{1}\left(\mathbb{F}_{q^{2}}\right)=(q \pm 1)^{2}$. The quadratic twist $E_{g^{3}}: y^{2}=x^{3}+g^{3}$ has $(q \mp 1)^{2}$ points over $\mathbb{F}_{q^{2}}$.

We consider $E_{g}: y^{2}=x^{3}+g$ over $\mathbb{F}_{q^{2}}$. Let $\phi: E_{g} \rightarrow E_{1}$ be the isomorphism $\phi(x, y)=(\alpha x, \beta y)$ where $\alpha \in \mathbb{F}_{q^{6}}$ and $\beta \in \mathbb{F}_{q^{4}}$ satisfy $\alpha^{3}=g$ and $\beta^{2}=g$. Let $\pi$ be the $q^{2}$-power Frobenius on $E_{g}$ and $\pi^{\prime}$ be the $q^{2}$-power Frobenius on $E_{1}$. Then $\pi^{\prime}=\mp[q]$ and so $\phi^{-1} \pi^{\prime} \phi=\mp[q]$. One can show that $\pi$ satisfies $T^{2} \pm q T+q^{2}=0$ and so $\# E_{g}\left(\mathbb{F}_{q^{2}}\right)=q^{2} \pm q+1$. It then follows that $E_{g^{2}}, E_{g^{4}}$ and $E_{g^{5}}$ also have $q^{2} \pm q+1$ points.

Lemma 8. Let $E$ be a supersingular elliptic curve over $\mathbb{F}_{q}$ (characteristic $>3$ ). Let $r \mid \# E\left(\mathbb{F}_{q}\right)$ with $r>3$ have security parameter $3 / 2$ or 3 . Then there is a distortion map $\phi$ on $E$, with easily computed inverse, such that if $P \in E\left(\mathbb{F}_{q}\right)[r]$ then $\phi(P) \in E\left(\mathbb{F}_{q^{3}}\right)[r]$ is a q-power Frobenius eigevector with eigenvalue $q$.

Proof. Let $\pi$ be the $q$-power Frobenius. Then security parameter $3 / 2$ or 3 implies that $\pi$ satisfies $\pi^{2} \pm q \pi+q=0$. Waterhouse [21] implies $q=p^{2 m}$ where $p \equiv 2$ (mod 3). Hence, by Lemma 6, $E$ is of the form $y^{2}=x^{3}+A$. Further, by Lemma 7 . $E$ is of the form $y^{2}=x^{3}+A$ where $A \in \mathbb{F}_{q^{2}}$ is not a cube.

We now define a distortion map on $E$. Note that $A$ may or may not be a square, but in either case $A / A^{q}$ is a square. Denote by $u$ a square root of $A / A^{q}$, and note that $u$ is not a cube. Let $\gamma \in \mathbb{F}_{q^{6}}$ satisfy $\gamma^{3}=u$ and note that $\gamma^{q^{2}}=\zeta_{3} \gamma$ for $\zeta_{3} \in \mathbb{F}_{q^{2}}$ such that $\zeta_{3}^{2}+\zeta_{3}+1=0$.

Define

$$
\phi(x, y)=\left(\gamma^{2} x^{q}, u y^{q}\right) .
$$

One can check that if $P \in E\left(\mathbb{F}_{q^{2}}\right)$ then $\phi(P) \in E\left(\mathbb{F}_{q^{6}}\right)$. Clearly $\phi$ and $\phi^{-1}$ are easily computed. 
It remains to prove that $\phi(P)$ is a Frobenius eigenvector, which we do in two stages. Let $P \in E\left(\mathbb{F}_{q^{2}}\right)[r]$, let $Q \in E\left(\mathbb{F}_{q^{6}}\right)[r]$ be a non-trivial point in the $q$-eigenspace of Frobenius, and let $\pi$ be the $q^{2}$-power Frobenius on $E$. One can show (see the full version of the paper for details) that

$$
\pi \phi(P)=\zeta_{3}^{2} \phi(P)
$$

where $\zeta_{3}(x, y)=\left(\zeta_{3} x, y\right)$ and $\zeta_{3}^{2}(x, y)=\zeta_{3} \circ \zeta_{3}(x, y)=\left(\zeta_{3}^{2} x, y\right)$. One can then show that

$$
\left(\pi^{2}+\pi+1\right)(\phi(P))=\left(\zeta_{3}^{2}+\zeta_{3}+1\right)(\phi(P))=0
$$

and so $\phi(P), \zeta_{3} \phi(P) \in\langle Q\rangle$ and $\phi(P)$ is a Frobenius eigenvector.

Theorem 3. Let $E$ be a supersingular elliptic curve over a finite field $\mathbb{F}_{q}$ suitable for pairing-based cryptography (i.e., with embedding degree $2 \leq k \leq 6$ and such that the class number of the field $\mathbb{Q}\left(\sqrt{t^{2}-4 q}\right)$ is at most $\left.\log (q)^{2}\right)$. Let $r>3$ be prime and coprime to $q$. Suppose that $r \mid \# E\left(\mathbb{F}_{q}\right)$ and that not all points in $E[r]$ are defined over $\mathbb{F}_{q}$. Let $k$ be the smallest positive integer such that $r \mid\left(q^{k}-1\right)$. Let $\pi$ be the q-power Frobenius map. Then $E[r]$ has a distortion eigenvector basis with respect to $F=\pi$.

Proof. Let $\pi$ be the $q$-power Frobenius. Since $r \mid \# E\left(\mathbb{F}_{q}\right)$ and $E[r] \nsubseteq E\left(\mathbb{F}_{q}\right)$ it follows from Balasubramanian and Koblitz 1 that $k>1$. Hence $q \not \equiv 1 \quad(\bmod r)$. Furthermore, $E[r]$ has a basis $\{P, Q\}$ such that $\pi(P)=P$ (i.e., $P \in E\left(\mathbb{F}_{q}\right)$ ) and $\pi(Q)=q Q$. It remains to prove the existence of a homomorphism $\phi:\langle P\rangle \rightarrow\langle Q\rangle$ for which $\phi$ and $\phi^{-1}$ can be computed in polynomial time.

In characteristic 2 , there are only finitely many $\mathbb{F}_{q}$-isomorphism classes of supersingular elliptic curves and we have $k \leq 4$ (see Menezes [18]). For applications we take $k=4$, in which case we may assume that $E$ is the elliptic curve

$$
E: y^{2}+y=x^{3}+x+b
$$

over $\mathbb{F}_{2^{m}}$ where $b=0$ or 1 and $m$ is odd. The field $\mathbb{F}_{2^{4 m}}$ has elements $s, t$ such that $s^{2}=s+1$ and $t^{2}=t+s$. Following 3 we consider the distortion map $\phi(x, y)=\left(x+s^{2}, y+s x+t\right)$. Note that $\phi$ and $\phi^{-1}$ are easily computed. It is immediate that if $P \in E\left(\mathbb{F}_{2^{m}}\right)$ then $\pi^{2}(\phi(P))=-\phi(P)$. Hence, $(P, \phi(P))$ is a distortion eigenvector base with respect to $F=\pi^{2}$.

To prove the result for $F=\pi$ suppose $\pi(\phi(P))=a P+b \phi(P)$ for some $0 \leq a, b<r$. Then $-\phi(P)=\pi(\pi(\phi(P)))=a(b+1) P+b^{2} \phi(P)$ and so $a(b+1) \equiv 0$ $(\bmod r)$ and $b^{2} \equiv-1 \quad(\bmod r)$. It follows that $a=0$ and $\phi(P)$ is an eigenvector for Frobenius (with eigenvalue $\pm q \quad(\bmod r)$ ).

In characteristic 3 , there are also only finitely many $\mathbb{F}_{q}$-isomorphism classes of supersingular elliptic curves and we have $k \leq 6$. For cryptographic applications we take $k=6$ and so we may assume that

$$
E: y^{2}=x^{3}-x+b
$$

over $\mathbb{F}_{3^{m}}$ where $b= \pm 1$ and $\operatorname{gcd}(m, 6)=1$. We consider the distortion map $\phi(x, y)=(\rho-x, \sigma y)$ where $\sigma, \rho \in \mathbb{F}_{3^{6}}$ satisfy $\sigma^{2}=-1$ and $\rho^{3}=\rho+b$. It 
is easy to check that if $P \in E\left(\mathbb{F}_{3^{m}}\right)$ and if $\pi$ is the $3^{m}$-power Frobenius then $\pi^{3}(\phi(P))=-\phi(P)$ so $(P, \phi(P))$ is a distortion eigenvector base with respect to $F=\pi^{3}$. The result also follows for $F=\pi$ using the same method as used in the case of characteristic 2 : write $\pi(\phi(P))=a P+b \phi(P)$, then $-\phi(P)=\pi^{3}(\phi(P))=$ $a\left(b^{2}+b+1\right) P+b^{3} \phi(P)$ and so $a=0$ and $b \equiv q \quad(\bmod r)$.

The case $k=3$ is of interest when $p>3$ satisfies $p \equiv 2 \quad(\bmod 3)$. The result is proved in Lemma 8 .

Finally, we consider the case $k=2$. Galbraith and Rotger [14 have given an algorithm to construct a distortion map $\phi$ for any supersingular elliptic curve $E$ over $\mathbb{F}_{q}$ where $q=p^{m}$ with $k=2$. The running time of the algorithm is polynomial in the running time of the CM method for constructing such an elliptic curve (and all known constructions of elliptic curves for pairing applications have small class number CM). Proposition 6.1 of 14 constructs the distortion map $\phi=\sqrt{-d}$ in $\operatorname{End}(E)$ where $d$ may be taken to be square-free. Then $\phi$ is an isogeny of degree $d$ which may be computed using Algorithm 1 of 14 . If $E$ has been constructed in polynomial time then we may assume that $d$ is bounded by a polynomial in $\log (p)$ and so this algorithm is polynomial time and it follows that $\phi$ may be computed in polynomial time.

Similarly, the dual isogeny $\hat{\phi}$ (see [19]) can be computed in polynomial time using an analogous algorithm. Recall that $\hat{\phi} \phi=[d]$.

Finally, the statement that $\phi(P)$ is a Frobenius eigenvector follows from the proof of Proposition 6.1 of [14. The $q$-power Frobenius lifts to the Galois element $\sigma$ in the proof, and $\phi$ lifts to an endomorphism $\Phi$ satisfying $\Phi^{\sigma}=-\Phi$. This implies $\pi \phi(P)=-\phi(P)=q \phi(P)$ as required.

A significant case not covered by the above theorem is the non-supersingular genus 2 curves proposed by Duursma and Kiyavash 9]. They consider the curves $y^{2}=x^{6}-a x^{3}+1$ and $y^{2}=x^{6}-a x^{3}-3$ over $\mathbb{F}_{p}($ where $p \equiv 2 \quad(\bmod 3))$. Define the isomorphism $\phi(x, y)=\left(\zeta_{3} x, y\right)$ where $\zeta_{3} \in \mathbb{F}_{p^{2}}$ is a primitive cube root of 1 . Note that $\phi^{2}+\phi+1=0$ in $\operatorname{End}(\operatorname{Jac}(C))$. Duursma and Kiyavash show that these curves satisfy the Yoshida conditions (see below). In particular, if $S \in \operatorname{Jac}(C)\left(\mathbb{F}_{p}\right)$ is a divisor class of order $r$ and if $F$ is the $p$-power Frobenius then $F(S)=S$ and $F(\phi(S))=-S-\phi(S)$.

Theorem 4. Let $C$ be one of the Duursma-Kiyavash curves and let notation be as above. Let $m=2^{-1}(\bmod r)$ and define $\phi^{\prime}=m+\phi$. Then $\left(S, \phi^{\prime}(S)\right)$ is a distortion eigenvector base.

Proof. It is easy to check (see Proposition 3below) that $F \phi^{\prime}(S)=-\phi^{\prime}(S)$. Hence $\left(S, \phi^{\prime}(S)\right)$ is an eigenvector base. Note also that $\phi^{\prime}$ is an efficiently computable group homomorphism.

To show that $\left(S, \phi^{\prime}(S)\right)$ is a distortion eigenvector base it remains to prove that there is an efficiently computable homomorphism $\phi^{\prime \prime}$ such that $\phi^{\prime \prime} \phi=d$ on $\langle S\rangle$. Consider the dual isogeny

$$
\widehat{m+\phi}=m+\widehat{\phi}
$$


Since $\widehat{\phi}=\phi^{2}$ we have

$$
(m+\widehat{\phi})(m+\phi)=m^{2}+m(\phi+\widehat{\phi})+\widehat{\phi} \phi=m^{2}-m+1 .
$$

Hence, define $d=\left(m^{2}-m+1\right) \quad(\bmod r)$ and $\phi^{\prime \prime}=m+\phi^{2}$ so that $\phi^{\prime \prime}$ is efficiently computable and $\phi^{\prime \prime} \phi^{\prime}=d$ on $\langle S\rangle$.

Corollary 4 can therefore be applied to deduce that VDP is equivalent to CDH for the Duursma-Kiyavash curves.

\section{Relation with the Yoshida Conditions}

Yoshida showed that $\mathrm{CDH} \leq \mathrm{VDP}$ when certain conditions on $G$ are satisfied. We have shown that $\mathrm{CDH} \leq \mathrm{VDP}$ when the group $G$ has an eigenvector base. In this section we show that Yoshida's result is a subcase of ours, by showing that if $G$ satisfies the Yoshida conditions then it has an eigenvector base. First we recall the conditions introduced by Yoshida in [23].

Definition 9. We say that $G$ satisfies the Yoshida conditions for $S \in G$ if there exist group isomorphisms $\phi, F: G \rightarrow G$ such that:

1. $\phi$ and $F$ can be computed in polynomial time;

2. $(S, \phi(S))$ is a base for $G$

3. Constants $\alpha_{1}, \alpha_{2}, \alpha_{3} \in \mathbb{Z} / r \mathbb{Z}$ are given, such that $\alpha_{1} \alpha_{2} \alpha_{3} \neq 0$ and

$$
F(S)=\alpha_{1} S, \quad F(\phi(S))=\alpha_{2} S+\alpha_{3} \phi(S) .
$$

We remark that we have been unable to find any groups satisfying the Yoshida conditions with $\alpha_{1}=\alpha_{3}$. Indeed, all known examples of groups satisfying the Yoshida conditions are when $G$ is a subgroup of a divisor class group of a curve over $\mathbb{F}_{q}, P$ is an element of prime order $r$ defined over the ground field $\mathbb{F}_{q}, F$ is a Frobenius map and $\phi$ is a non- $\mathbb{F}_{q}$-rational endomorphism of the curve. It follows that $\alpha_{1}=1$.

Proposition 3. If $G$ satisfies the Yoshida conditions for $S$ then one can calculate $T \in G$ such that $(S, T)$ is an eigenvector base.

Proof. Suppose $S, F, \phi$ satisfy the Yoshida conditions.

First suppose that $\alpha_{1} \neq \alpha_{3}$. Let $m=\left(\alpha_{3}-\alpha_{1}\right)^{-1} \alpha_{2} \quad(\bmod r)$ and let $\phi^{\prime}=$ $m+\phi$. Then

$$
\begin{aligned}
F\left(\phi^{\prime}(S)\right) & =F(m S+\phi(S))=\alpha_{1} m S+\alpha_{2} S+\alpha_{3} \phi(S) \\
& =\left(\alpha_{1} m+\alpha_{2}-\alpha_{3} m\right) S+\alpha_{3} \phi^{\prime}(S) \\
& =\alpha_{3} \phi^{\prime}(S) .
\end{aligned}
$$

It follows that $\left(S, \phi^{\prime}(S)\right)$ is an eigenvector base. 
Now we deal with the case $\alpha_{1}=\alpha_{3}$ (which possibly never occurs in practice). Set $\theta=\alpha_{2}^{-1} \quad(\bmod r), \gamma=\alpha_{2}^{-1} \alpha_{1} \quad(\bmod r)$ and define

$$
\psi(R)=\theta F(R)-\gamma R
$$

for $R \in G$. It follows that

$$
\psi(S)=\left(\theta \alpha_{1}-\gamma\right) S=0
$$

and

$$
\psi(\phi(S))=\theta \alpha_{2} S+\left(\theta \alpha_{3}-\gamma\right) \phi(S)=S .
$$

Consequently, if we take $\psi^{\prime}=\phi \circ \psi$ we get that $\psi^{\prime}(S)=0$ and $\psi^{\prime}(\phi(S))=\phi(S)$. That is, $\psi^{\prime}$ is the projection on $\langle\phi(S)\rangle$ w.r.t. the base $(S, \phi(S))$. So $R-\psi^{\prime}(R)$ is the projection of $R$ on $\langle S\rangle$ w.r.t. the base $(S, \phi(S))$. Consequently if we take $F^{\prime}(R)=\lambda_{2} \psi^{\prime}(R)+\lambda_{1}\left(R-\psi^{\prime}(R)\right)$ for any distinct non-zero $\lambda_{1}, \lambda_{2} \in \mathbb{Z} / r \mathbb{Z}$ it easily follows that $(S, \phi(S))$ is an eigenvector base for $F^{\prime}$ and $\phi$.

Note that in many cases the above proof yields a distortion eigenvector base. However, we cannot prove this in all cases since the Yoshida conditions contain no requirement that the dual isogeny of $\phi$ be efficiently computable.

For completeness we show how to transfrom a distortion eigenvector base to satisfy the Yoshida conditions.

Lemma 9. Let $G$ be a group with homomorphisms $\phi, F$ and an eigenvector base $(S, \phi(S))$. Let $\phi^{\prime}=1+\phi$. Then $G$ together with $\phi^{\prime}, F$ satisfies the Yoshida conditions.

Proof. Clearly the first two Yoshida conditions hold. For the third, one checks that

$$
F\left(\phi^{\prime}(S)\right)=F(S+\phi(S))=\lambda_{1} S+\lambda_{2} \phi(S)=\left(\lambda_{1}-\lambda_{2}\right) P+\lambda_{2} \phi^{\prime}(P)
$$

which completes the proof

Corollary 5. Let $E$ be any supersingular elliptic curve used in practice as above. Then one can construct a triple $(P, F, \phi)$ satisfying the Yoshida conditions.

\section{$5 \quad$ Non-cyclic Groups}

The VDP is defined for any group $G$ of exponent $r$ and order $r^{2}$. In this section we very briefly recall some non-cyclic groups which might be suitable for cryptography. Recall that the main groups of interest in discrete-logarithm based cryptography are the multiplicative group of a finite field (which is always cyclic) and elliptic curves or divisor class groups of curves (which can be non-cyclic). For background on elliptic curves in cryptography (and pairings) see 448.

1. Direct products $G=G_{1} \times G_{2}$ where $G_{1}, G_{2}$ are cyclic subgroups of finite fields, elliptic curves or divisor class groups. 
2. Elliptic curves $E$ over $\mathbb{F}_{q}$ such that the group of points of order $r$ (called the $r$-torsion subgroup) is defined over a small degree extension $\mathbb{F}_{q^{k}}$. Such curves are automatically 'pairing-friendly'. There are two cases:

(a) Supersingular curves.

(b) Ordinary curves. There are many methods to generate pairing-friendly ordinary curves (see [1] for a survey).

3. Subgroups of exponent $r$ and order $r^{2}$ of the divisor class group of a curve of genus $g \geq 2$ over $\mathbb{F}_{q^{k}}$. In this case, the full $r$-torsion is not necessarily defined over $\mathbb{F}_{q^{k}}$ and so the divisor class group is not necessarily pairingfriendly. Again, there are two cases.

(a) Supersingular. These curves are necessarily pairing-friendly. There are many examples of supersingular hyperelliptic curves given in the literature (see 13]).

(b) Non-supersingular. For example the curves with complex multiplication presented by Duursma and Kiyavash [9].

4. The subgroup of order $r^{2}$ in $(\mathbb{Z} / n \mathbb{Z})^{*}$ where $n=p q$ is a product of two primes such that $r \mid(p-1)$ and $r \mid(q-1)$. Care must be taken that $r$ is not too large, or else it is easy to factor $n$ (see McKee and Pinch [17]).

This case has a very different flavour to the other groups described above, and the methods of the paper do not seem to apply in this case.

Note that not all of the above groups will necessarily have an eigenvector base.

\section{Generalised Discrete Logarithm Problems}

We have proved that VDP is equivalent to $\mathrm{CDH}$ in a cyclic group for all examples proposed in the literature. But one might consider VDP in a more general context where distortion maps $\phi$ are not available. Hence we give some results relating VDP to generalisations of the discrete logarithm problem. As always, $G$ denotes a group of order $r^{2}$ and exponent $r$ where $r$ is prime. Due to lack of space, many of the proofs in this section have removed; they can be found in the full version of the paper.

We recall the discrete logarithm problem $\left(\mathrm{DLP}_{G_{1}}\right)$ for a cyclic group $G_{1}$ : Given $P, Q \in G_{1}$, compute an integer $a$ (if it exists) such that $Q=a P$. The discrete logarithm problem has been generalized by many authors in different ways. For example, if $G_{1}$ is a cyclic group of prime order and $P_{1}, P_{2} \in G_{1}$ then Brands [6] defined the representation problem: Given $Q \in G_{1}$ find $(a, b)$ such that $Q=a P_{1}+b P_{2}$. It is easy to show that the the representation problem in the cyclic group $G_{1}$ is equivalent to the DLP in $G_{1}$.

For groups $G$ of exponent $r$ and order $r^{2}$ we define the following generalisation of the discrete logarithm problem.

Definition 10. The computational problem 2-DL is: Given $P_{1}, P_{2}, Q \in G$ such that $G=\left\langle P_{1}, P_{2}\right\rangle$ compute a pair of integers $(a, b)$ such that $Q=a P_{1}+b P_{2}$.

The following three results are straightforward. 
Lemma 10. The computational problem 2-DL is random self-reducible.

Lemma 11. Let $G_{1}$ be a cyclic subgroup of $G$. Then $D L P_{G_{1}} \leq 2-D L$.

Theorem 5. Let $G$ be as above. Then $V D P \leq 2-D L$.

The computational problems VDP and 2-DL are both defined for non-cyclic groups. Computational problems in non-cyclic groups have not been studied as closely as those in cyclic groups. The remainder of this section relates the 2DL problem in non-cyclic groups to discrete logarithm problems in one or more cyclic groups.

Let $G_{1}, G_{2}$ be cyclic groups of order $r$. We say that two group homomorphisms $\psi_{i}: G \rightarrow G_{i}$, for $i=1,2$, are independent if ker $\psi_{1} \cap \operatorname{ker} \psi_{2}=\{0\}$. An example of independent group homomorphisms are the projection maps in the proof of Proposition 1 .

Theorem 6. Let $G$ and $G_{1}$ be as above and suppose there are two independent group homomorphisms $\psi_{1}, \psi_{2}: G \rightarrow G_{1}$ which can be computed in polynomial time. Then 2-DL is equivalent to $D L P_{G_{1}}$.

This result is a special case of the following.

Theorem 7. Let $G$ be as above and let $G_{1}, G_{2}$ be cyclic groups of order $r$. Suppose there are two independent group homomorphisms $\psi_{i}: G \rightarrow G_{i}$ for $i=1,2$ which can be computed in polynomial time. Then 2-DL is equivalent to $\left(D L P_{G_{1}}\right.$ and $\left.D L P_{G_{2}}\right)$.

Proof. It is trivial from Lemma 11 that $\left(\mathrm{DLP}_{G_{1}}\right.$ and $\left.\mathrm{DLP}_{G_{2}}\right) \leq 2$-DL. One can prove the opposite using essentially the same ideas as those used in the proof of Theorem 1 .

Corollary 6. If $G$ has an eigenvector base $(S, T)$ then 2-DL is equivalent to $\left(D L P_{\langle S\rangle}\right.$ and $\left.D L P_{\langle T\rangle}\right)$.

Corollary 7. Let $G$ be a group which has a distortion eigenvector base $(S, T)$. Let $G_{1}=\langle S\rangle$. Then 2-DL is equivalent to $D L P_{G_{1}}$.

Proof. We let $\psi_{1}$ be as in the proof of Proposition 1 and let $\psi_{2}(Q)=\psi_{1}(\phi(Q))$. One can check that these are independent homomorphisms to $\langle S\rangle$, and so the result follows from Theorem 6 .

Direct products (case 1 of Section 5) are easy to handle.

Corollary 8. Let $G$ be a direct product of two cyclic groups $G_{1}, G_{2}$ of prime order $r$. Then 2-DL $\leq\left(D L P_{G_{1}}\right.$ and $\left.D L P_{G_{2}}\right)$.

On ordinary pairing-friendly elliptic curves (i.e., case 2(b) of Section 5) we do not have distortion maps and so it is not possible to have a distortion eigenvector base. We now state the obvious fact that the 2-DL can be reduced to the DLP in a finite field using pairings. 
Theorem 8. Let $G$ be a subgroup of $E\left(\mathbb{F}_{q^{k}}\right)$ of exponent $r$ and order $r^{2}$. Then $r \mid\left(q^{k}-1\right)$. Let $G_{1}$ be the subgroup of $r$-th roots of unity in $\mathbb{F}_{q^{*}}^{*}$. Then 2-DL $\leq$ $D L P_{G_{1}}$.

In the ordinary genus 2 case (again, case 3(b) of Section 5) there is another way to potentially attack the 2-DL. One natural approach to constructing a curve $C$ over $\mathbb{F}_{q}$ whose Jacobian has non-cyclic group order is to choose $C$ such that there are rational maps $\psi_{i}: C \rightarrow E_{i}$ (for $i=1,2$ ) over $\mathbb{F}_{q}$ where $E_{i}$ are elliptic curves over $\mathbb{F}_{q}$. Then the Jacobian of $C$ is isogenous over $\mathbb{F}_{q}$ to $E_{1} \times E_{2}$ and if $r \mid \# E_{i}\left(\mathbb{F}_{q}\right)$ for $i=1,2$ then $r^{2}$ divides the order of $\operatorname{Jac}(C)\left(\mathbb{F}_{q}\right)$. This approach was used by Duursma and Kiyavash 9. Since the rational maps $\psi_{i}$ induce explicit isogenies

$$
\psi_{i}: \operatorname{Jac}(C)\left(\mathbb{F}_{q}\right) \rightarrow E_{i}\left(\mathbb{F}_{q}\right)
$$

for $i=1,2$ one can apply Theorem 7 to reduce the 2-DL to two DLPs in cyclic groups.

\section{Conclusion}

We present a thorough analysis of the vector decomposition problem (VDP). We have shown that, for all the supersingular elliptic curves which could be used in practice, VDP is equivalent to $\mathrm{CDH}$ in a cyclic group. We have also related VDP to various co-CDH problems and a generalised discrete logarithm problem 2-DL which in turn is often related to discrete logarithm problems in cyclic groups.

\section{Acknowledgements}

We thank Iwan Duursma, Seung-Kook Park, Maura Paterson and a number of anonymous referees for helpful comments on a much earlier draft of the paper. Takakazu Satoh is thanked for proof reading. Galbraith also thanks the Fields Institute in Toronto for providing a stimulating research environment during some of this research and EPSRC Research Grant EP/D069904/1 for financial support.

\section{References}

1. Balasubramanian, R., Koblitz, N.: The improbability that an elliptic curve has subexponential discrete log problem under the Menezes-Okamoto-Vanstone algorithm. J. Cryptology 11(2), 141-145 (1998)

2. Bao, F., Deng, R.H., Zhu, H.: Variations of Diffie-Hellman Problem. In: Qing, S., Gollmann, D., Zhou, J. (eds.) ICICS 2003. LNCS, vol. 2836, pp. 301-312. Springer, Heidelberg (2003)

3. Barreto, P.S.L.M., Kim, H.Y., Lynn, B., Scott, M.: Efficient algorithms for pairingbased cryptosystems. In: Yung, M. (ed.) CRYPTO 2002. LNCS, vol. 2442, pp. 354-368. Springer, Heidelberg (2002) 
4. Blake, I., Seroussi, G., Smart, N.P. (eds.): Advances in elliptic curve cryptography. Cambridge University Press, Cambridge (2005)

5. Boneh, D., Lynn, B., Shacham, H.: Short signatures from the Weil pairing. Journal of Cryptology 7, 297-319 (2004)

6. Brands, S.: An efficient off-line electronic cash system based on the representation problem, CWI Technical Report CS-R9323 (1993)

7. Charles, D.: On the existence of distortion maps on ordinary elliptic curves, arXiv:math/0603724 (2006)

8. Cohen, H., Frey, G. (eds.): Handbook of elliptic and hyperelliptic curve cryptography. CRC Press, Boca Raton (2006)

9. Duursma, I., Kiyavash, N.: The vector decomposition problem for elliptic and hyperelliptic curves. J. Ramanujan Math. Soc. 20(1), 59-76 (2005)

10. Duursma, I.M., Park, S.K.: ElGamal type signature schemes for n-dimensional vector spaces, eprint 2006/311

11. Freeman, D., Scott, M., Teske, E.: A taxonomy of pairing-friendly elliptic curves (2006)

12. Frey, G., Rück, H.-G.: A remark concerning $m$-divisibility and the discrete logarithm in the divisor class group of curves. Math. Comp. 62, 865-874 (1994)

13. Galbraith, S.D.: Supersingular curves in cryptography. In: Boyd, C. (ed.) ASIACRYPT 2001. LNCS, vol. 2248, pp. 495-513. Springer, Heidelberg (2001)

14. Galbraith, S.D., Rotger, V.: Easy decision Diffie-Hellman groups. LMS J. Comput. Math. 7, 201-218 (2004)

15. Maurer, U.: Towards the equivalence of breaking the Diffie-Hellman protocol and computing discrete logarithms. In: Desmedt, Y.G. (ed.) CRYPTO 1994. LNCS, vol. 839, pp. 271-281. Springer, Heidelberg (1994)

16. Maurer, U., Wolf, S.: The relationship between breaking the Diffie-Hellman protocol and computing discrete logarithms. SIAM Journal on Computing 28(5), 1689 1721 (1999)

17. McKee, J.F., Pinch, R.G.E.: Further attacks on server-aided RSA cryptosystems, unpublished manuscript (1998)

18. Menezes, A.J.: Elliptic curve public key cryptosystems. Springer, Heidelberg (1993)

19. Silverman, J.H.: The arithmetic of elliptic curves. Springer, Heidelberg (1986)

20. Verheul, E.R.: Evidence that XTR Is More Secure than Supersingular Elliptic Curve Cryptosystems. J. Cryptology 17(4), 277-296 (2004)

21. Waterhouse, W.C.: Abelian varieties over finite fields. Annales Scientifiques de l'École Normale Supérieure 4 (1969)

22. Yoshida, M., Mitsunari, S., Fujiwara, T.: Vector decomposition problem and the trapdoor inseparable multiplex transmission scheme based the problem. In: Proceedings of the 2003 Symposium on Cryptography and Information Security (SCIS), pp. 491-496 (2003)

23. Yoshida, M.: Inseparable multiplex transmission using the pairing on elliptic curves and its application to watermarking. In: Proc. Fifth Conference on Algebraic Geometry, Number Theory, Coding Theory and Cryptography, University of Tokyo (2003), http://www.math.uiuc.edu/ duursma/pub/yoshida_paper.pdf 